\title{
NRSfM-Flow: Recovering Non-Rigid Scene Flow from Monocular Image Sequences
}

\author{
Vladislav Golyanik ${ }^{1,2}$ \\ http://av.dfki.de/members/golyanik/ \\ Aman Shankar Mathur ${ }^{1}$ \\ asmathur@rhrk.uni-kl.de \\ Didier Stricker ${ }^{1,2}$ \\ http://av.dfki.de/members/stricker/
}

\author{
${ }^{1}$ Department of Computer Science \\ University of Kaiserslautern \\ Kaiserslautern, Germany \\ ${ }^{2}$ Department Augmented Vision \\ German Research Center for Artificial \\ Intelligence (DFKI GmbH) \\ Kaiserslautern, Germany
}

Recovery of scene flow (a dense 3D velocity vector field) of a dynamic scene from monocular image sequences is an emerging field in computer vision. Being sensitive to occlusions, existing Monocular Scene Flow (MSF) methods are either limited in handling non-rigid deformations [5], or make strong assumptions on scene [2] and camera motion [1]. To overcome these limitations, we propose a framework for MSF estimation based on Non-Rigid Structure from Motion (NRSfM) [4] techniques NRSfM-Flow. In the continuous domain, relation between a shape $\mathbf{S}(\mathbf{p}, t)$, camera motion $\mathbf{R}(t)$ and scene flow $\Theta(\mathbf{p}, t)$ can be expressed as

$$
\Theta(\mathbf{p}, t)=\frac{\partial \mathbf{R}(t)}{\partial t} \mathbf{S}(\mathbf{p}, t)+\mathbf{R}(t) \frac{\partial \mathbf{S}(\mathbf{p}, t)}{\partial t} .
$$

To enhance reconstruction accuracy and speedup computations, two preprocessing steps are proposed - Translation Resolution (TR) and Redundancy Removal (RR). With TR, translation of the scene is resolved using a sparse point tracker. Using RR, frames with insufficient diversity are dropped according to the criterion

$$
\left\|\int_{\hat{\Psi}} \int_{t_{a}}^{t_{b}} \mathbf{\Xi}(\mathbf{v}, t) d t d \hat{\mathbf{v}}\right\|_{2} \geq \varepsilon
$$

where $\boldsymbol{\Xi}(\mathbf{v}, t)$ is a continuous optical flow function, $\hat{\mathbf{v}} \in \hat{\Psi} \subset \mathbb{R}^{2}$ are $2 \mathrm{D}$ points observed at a reference time $\tau$, and $\varepsilon$ is a scalar threshold.

Our approach can handle long image sequences with non-rigid deformations and self-occlusions, with no strong assumptions such as a known camera motion. Performance is demonstrated on several synthetic and real image sequences (see Fig. 1 for an example). With this paper we hope, on the one hand, to draw attention to modelbased approaches for MSF estimation and, on the other, to highlight importance of the differential interpretation of the NRSfM problem.

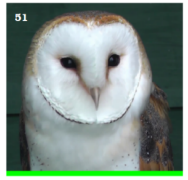

(a)

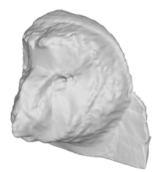

(d)

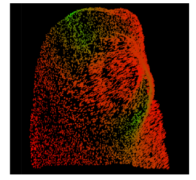

(b)

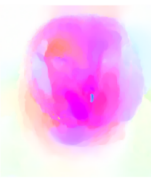

(e)

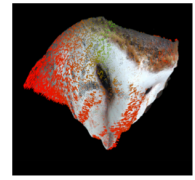

(c)

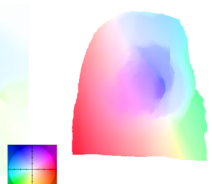

(f)
Figure 1: Experimental results on the barn owl sequence [3]: (a) frame 51; (b) scene flow between frames 51 and 52; (c) geometry + scene flow; (d) shaded geometry (Poisson) from a novel viewpoint; (e) optical flow between frames 51 and 52; (f) projection of the 3D motion field into the image plane.

[1] N. Birkbeck, D. Cobzaş, and M. Jägersand. Depth and scene flow from a single moving camera. In $3 D$ Data Processing Visualization and Transmission (3DPVT), 2010.

[2] N. Birkbeck, D. Cobzaş, and M. Jägersand. Basis constrained $3 \mathrm{~d}$ scene flow on a dynamic proxy. In International Conference on Computer Vision (ICCV), pages 1967-1974, 2011.

[3] P. Dinning. Barn Owl at Screech Owl Sanctuary. https: //www. youtube. com/watch? $\mathrm{v}=x \operatorname{mou} 8 \mathrm{t}-\mathrm{DHh} 0,2014$. [online; accessed on 12.05.2016; usage rights obtained from the author].

[4] R. Garg, A. Roussos, and L. Agapito. Dense variational reconstruction of non-rigid surfaces from monocular video. In Computer Vision and Pattern Recognition (CVPR), pages 1272-1279, 2013.

[5] D. Xiao, Q. Yang, B. Yang, and W. Wei. Monocular scene flow estimation via variational method. Multimedia Tools and Applications (An International Journal), pages 1-23, 2015. 